Wolfram Horstmann*

\title{
Are Academic Libraries Changing Fast Enough?
}

https://doi.org/10.1515/bfp-2018-0061

\begin{abstract}
Academic libraries are under a severe pressure of transforming towards a novel form of information organization. Book circulation, learning space and licensing digital content continue to be core services. But the sciences and the humanities are increasingly requesting support for their novel publishing activities. Open Access in science or digital editions in the humanities, and data-intensive operations in research, e.g. data management plans, research software support or data curation and preservation services become mission critical. Thus, in order to stay the central partner for academic information within the institution, libraries need to change. But how fast could libraries possibly change, if the existing services were also to be continued, particularly considering that budget increases are rare? Experiences at the State and University Library Göttingen (SUB) shall elucidate opportunities and challenges.
\end{abstract}

Keywords: Academic library; change management; digital transformation; scholarly communication

\section{Ändern sich wissenschaftliche Bibliotheken schnell ge- nug?}

Zusammenfassung: Akademische Bibliotheken stehen unter dem starken Druck, sich zu einer neuen Form der Informationsorganisation zu entwickeln. Die Verbreitung von Büchern, der Lernraum und die Lizenzierung digitaler Inhalte sind weiterhin Kerndienstleistungen. Aber die Naturwissenschaften und die Geisteswissenschaften fordern zunehmend Unterstützung für neue Publikationsformate und Kommunikationsformen. Open Access in den Naturwissenschaften oder digitalen Editionen in den Geisteswissenschaften sowie datenintensive Aktivitäten in der Forschung, z.B. Datenmanagementpläne, Unterstützung von Forschungssoftware oder Datenkuration und -archivierung werden geschäftskritisch. Um also der zentrale Partner für akademische Informationen innerhalb der Institution zu bleiben, müssen sich die Bibliotheken verändern. Aber wie schnell könnten sich die Bibliotheken verändern, wenn die bestehenden Dienstleistungen auch weitergeführt werden sollten, zumal eine Erhöhung des Budgets

*Kontaktperson: Prof. Dr. Wolfram Horstmann, horstmann@sub.uni-goettingen.de selten ist? Erfahrungen an der Staats- und Universitätsbibliothek Göttingen (SUB) zeigen Chancen und Herausforderungen auf.

Schlüsselwörter: Wissenschaftliche Bibliotheken; Change Management; Innovation; Bibliotheksstrategie

\section{Introduction}

Innovation in scholarly communication is rapid and disruptive. Dozens of new services have been introduced over the last years that are crucial in research but not regularly supported by librarians. ${ }^{1}$ Alone, for the transformation from subscription models to Open Access in the STM journal market, more than ten substantial new services can be identified. ${ }^{2}$ Research data management is increasingly considered as a major new field to be taken on by libraries. ${ }^{3}$ And the pipeline of global technology for innovation is full, with sometimes erratic, disruptive and unpredictable implications for libraries. ${ }^{4}$ The questions, thus, are:

1. Do libraries transform fast enough and have a sufficiently precise model for strategically steering transformation?

2. Do libraries have sufficient transformation capacity to respond to innovations in research, teaching and learning?

Common sense considerations about the transformation capacity of an academic library provide starting points for developing a strategic plan. For the sake of parsimony (and realism), it is assumed that recurrent budgets of libraries to add new tasks in their service portfolio by appointing additional staff and contracting additional services cannot be increased. For the sake of simplicity, it is assumed that tasks are equal to Full-Time-Equivalents (FTE) even though tasks could also be outsourced and would occur as non-staff costs.

For a given operational budget, the pace of library transformation ( $T$ ) can then be determined by the ratio between tasks being reduced and tasks being added. Re-

1 Kramer and Bosman (2016).

2 Horstmann (2017).

3 Tenopir et al. (2015, 2017).

4 Prinsloo und Van Deventer (2017). 


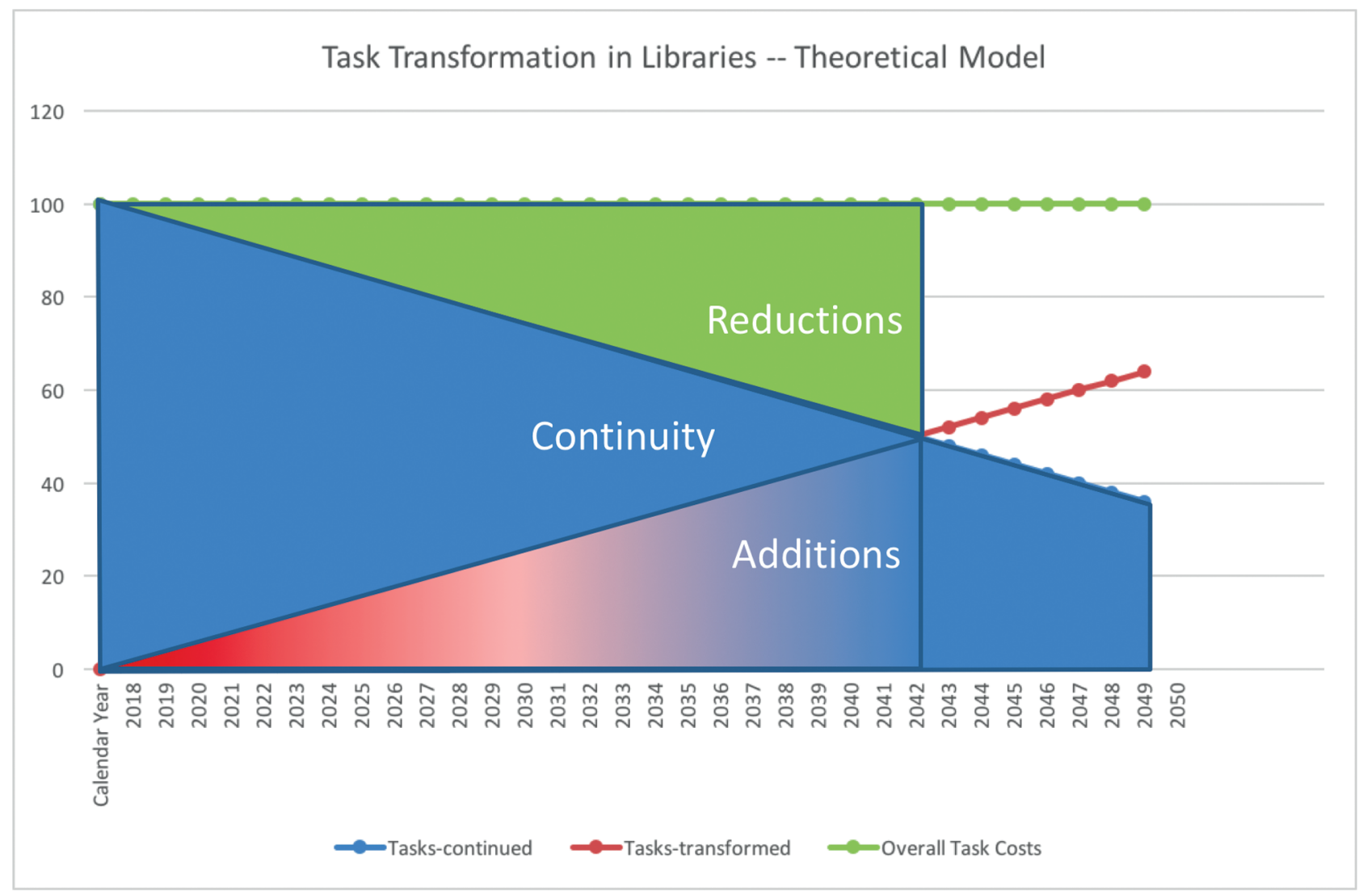

Fig. 1: Task transformation in libraries - theoretical model ${ }^{5}$

ductions $(R)$ as well as Additions (A) are dependent on at least four factors (T=R/A; with parameters a, b, c, d):

a. Appointment Capacity: The ability to appoint new staff for taking on new tasks

b. Re-Skilling Capacity: The ability to train new staff for taking on new tasks

c. Task Efficiencies: The amount of existing tasks being compressed, i.e. using less FTE or reducing contract costs

d. Task Cancellations: The amount of tasks being discontinued

These simple considerations allow a projection of service transformation in the library: if an average $2 \%$ change per year is implemented, it takes 25 years to transform $50 \%$ of tasks (see Figure 1). In other words, half of the library's service capacity can be kept for the continuation of exist-

5 In a scenario that assumes 100 cost units for tasks in a library, say 100 FTEs, it would take 25 years to transform $50 \%$ of the tasks, if 2 FTE are added each year and 2 FTE are reduced each year. The red-to-blue colour transition in the summative additions indicate that additions become part of the continuity and have to be counted on top of the continuity area. ing services such as book circulation, learning space of digital content licensing, while $50 \%$ can be allocated for new tasks such as Open Access or Research Data Management.

In the arbitrary example of introducing a campus reference service for research data management with 1 FTE in the library, the following steps would have to be taken: (i) a post from a staff member going into pension or leaving the organization would have to be free, (ii) a new person could be appointed or an existing staff member takes over, (iii) former tasks of the leaving or re-positioned staff member are either discontinued or efficiencies are found by distributing the tasks to other staff members.

Two obvious measures to boost transformation capacity can be applied, both of which already strategically or pragmatically applied by many libraries: Additional grants such as temporary support from the institution, research grants or philanthropic funding and pre-filling of positions that will become available. In the abovementioned example, the library would receive funding from the University to establish the campus reference service as pilot but is asked to make the post permanent after 2 years. Since the library does currently not have a free position, a position becoming free in 2 years is pre-filled and other tasks are to 
be cancelled or efficiencies are to be found. Accordingly, the number of FTE would be 101 for two years.

The abovementioned rationales are applied to the Göttingen State and University Library (SUB), to discuss questions (1) and (2). It is not intended to provide a mathematical sound model at this stage but rather demonstrate the considerations to be taken into account and the data to be gathered to merely enter the stage of strategic planning.

\section{About the State and University Library Göttingen (SUB)}

The SUB is not the typical example of an academic library but a rather large and complex exemplar. However, the considerations addressed in this text should be robust, i.e. transferrable to other academic libraries, if figures were simply put in a relative form (percentages).

The SUB is currently undergoing an unprecedented transformation from a local service organization to a global player in academic information. It is today not only a massively digitized enterprise that provides, on average, one service each second of the year to 44,000 local users and much wider a global audience - it also engages in research in ways not yet to be found regularly in libraries. Just as examples, librarians at the SUB develop data acquisition systems for biodiversity research in the Indonesian rainforest or consult university strategy with data-intensive research performance analytics. With its service transformation and innovation capacity and an average annual project budget of ca $5 \mathrm{~m} €$, the SUB has added structures resembling an engineering company and a research department on top of its traditional functions. Through its engagement in research and development (R\&D) projects as well as science policy work, the SUB has built a network of formal partnerships with 136 partners in 26 countries (see Figure 2).

In the size of its collections, the SUB compares to large academic libraries in the US, e.g. Cornell, Princeton, Stanford or Yale, and ranges, in terms of books, among the three largest University Libraries in Germany. It has extensive special collections that attract scholars and citizens around the world, not least with its unique vellum edition of the Gutenberg-Bible, which has the UNESCO-status "Memory of the World". In absence of a German national library before the $19^{\text {th }}$ and $20^{\text {th }}$ century, large libraries in Germany share responsibilities for maintaining and developing collections in certain temporal periods as a distributed national library system, in which the SUB takes care of the $18^{\text {th }}$ century.

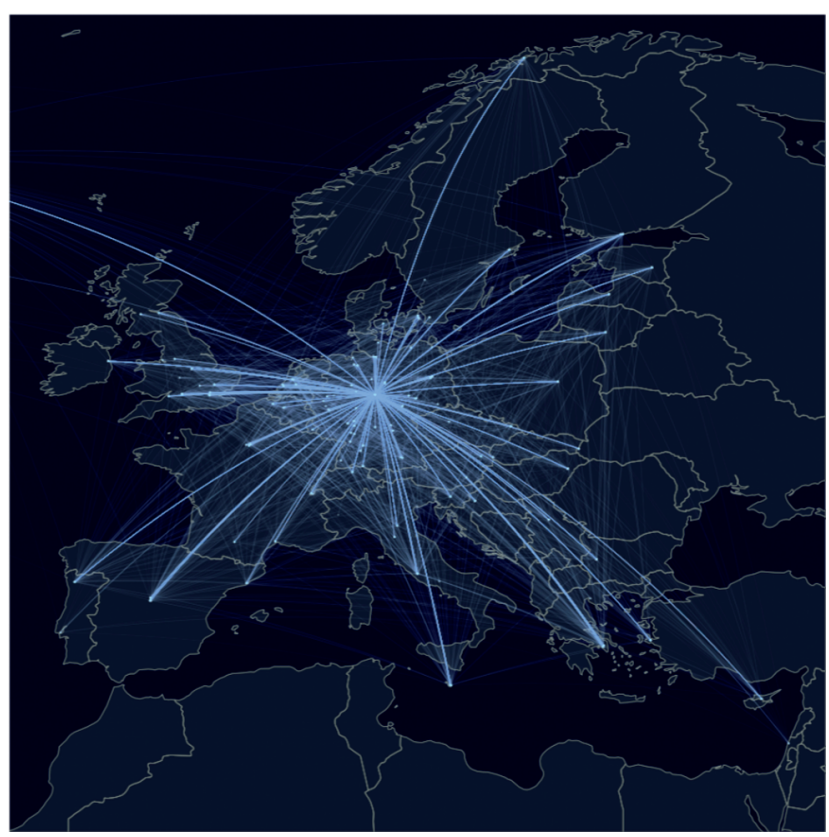

Fig. 2: European part of the international network of formal partnerships with academic institutions currently operated by the SUB (visualization provided by Najko Jahn)

\section{Appointment Capacity}

In a staff-centric approach to service transformation, the ability to appoint new staff is a central element. Re-Skilling (see below) is equally important but may fail when innovation is rapid or disruptive and re-skilling is not fast or efficient enough. As organizations dedicated to conservative functions and often being in a public system, libraries work on the basis of permanent posts and rarely cancel contracts with employees due to re-structuring as it is common practice in industry. Long-term planning on appointment capacity, thus, is crucial. At the SUB, an analysis of appointment capacity showed that a statistical assumption on average staff numbers and pensions is not sufficient. Rather a detailed account is necessary to detect irregularities and deviations from statistical assumptions (Figure 2). In order to precisely and responsibly calculate these projections, there is no alternative to an empirical approach, i.e. carefully assessing each individual staff member to account for personal conditions and choices such pension age or part-time work. Obviously, these data are also needed to monitor pre-filling practice.

The data in this example (see Figure 3) show that appointment capacity is unusually low until 2020 and depleted by pre-filling. These calculations were also used to reach a long-term agreement with university and staff committees for a transparent and generalized plan on a 


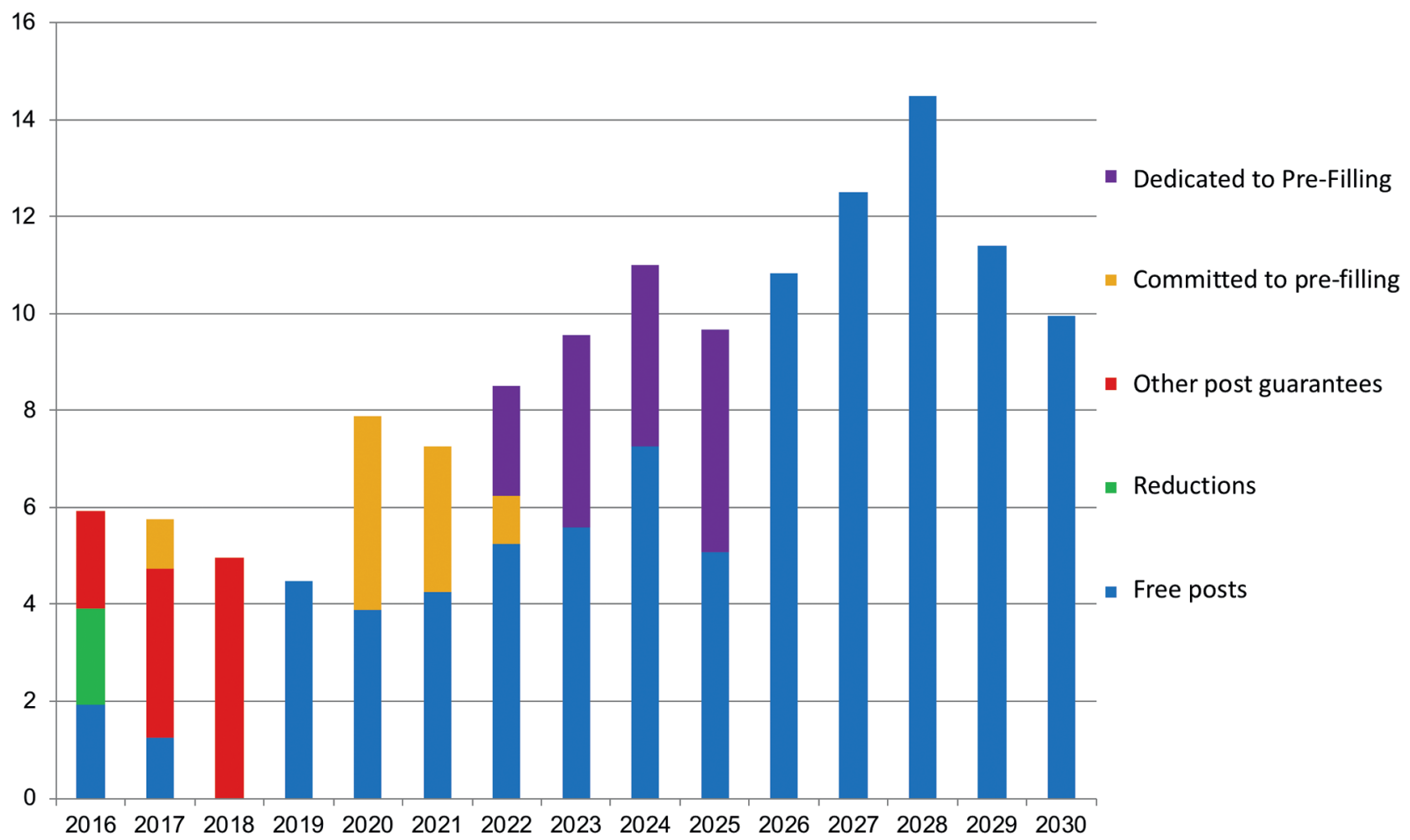

Fig. 3: Snapshot of appointment capacity at the SUB ( $x$-axis: years; $y$-axis: FTE)

percentage of posts that can be used for pre-filling in each given year (yellow and purple bars in Figure 3).

Pre-filling was then used to implement a first wave of new appointments to increase the analytical capacity of the SUB by 5 positions (see Figure 3, yellow bars):

1. Legal Analyst - IPR and Licensing (Exploitation, open rights)

2. Data Analyst - Scholarly Information (Bibliometrics, Information Market)

3. Data Analyst - Spatial and Geospatial Data (GIS as cross-cutting data type)

4. Data Analyst - Language and Text Data (Corpus linguistics und 'Mining' as a Service)

5. Process Analyst - ILS, Cataloguing, Search (Refactoring the 'Enterprise'-Systems)

Financing pre-filling requires investments or "bridge funding": the years between appointment and integration into the regular budget have to be covered. One possible way to implement bridge funding is to build up savings from the regular budgets, for example, by implementing top-sliced savings in the organization. However, these savings are equivalent of additional reductions of tasks, which is usually limited by the demand of service continuity. Also, if tasks could be reduced, pre-filling is somewhat circular because reductions could also be directly used for new appoint- ments. Another way to finance pre-filling is to get additional grants. R\&D grants, however, are usually dedicated to certain project tasks and, only if the research grant matched precisely the requirement of the post to be pre-filled appointments of adequate staff members will be possible. Structural grants of the institution (or government) that are dedicated to service transformation are the optimal source of bridge funding. This would require, for example, to submit a strategic proposal how exactly the library has to change and what dedicated transformation budget is requested for this (see also below: Discussion). Such a plan could be regarded to be equivalent to the use of investment funds in industry.

\section{Re-Skilling Capacity}

The ability of staff to take on a new task is another crucial element in service transformation. If efficiencies are found and a staff member has the possibility to work on new tasks, the question still is whether this person is actually qualified to do so. Ongoing training and qualification programs in the library are, of course, the first choice for staff development. They have been discussed intensively ${ }^{6}$ and

6 Julien et al. (2018). 
shall not further be addressed in this text. Rather, the possibilities of training-on-the-job shall be considered in more detail. Organizational change, i.e. finding efficiencies or reducing tasks and taking on new tasks, is a local and gradual process. Eventually, change is about individuals who come to work every day, perform certain tasks and, at the point of change, are expected to leave one task (or spend less time on it) and perform another task instead. It is hardly imaginable, that management is specifying, implementing and monitoring each individual change process. Thus, a localized change mechanism has to be found. Strategic plans of an organization can provide cornerstones for change and, most importantly, implementation plans will provide opportunities to define change processes.

At the SUB, a concept of internal projects is being used to plan and implement change locally and gradually. To ramp up the traditional service operations based on operational funding, a systematic annual strategy process was introduced in 2014. In an open call, staff and senior management submit prospective internal implementation projects in the summer of a given year to jointly plan projects for the next year. Each project has to be referenced to the strategic goals of the SUB. A condition for these projects is that they are cost neutral, i.e. there is no additional funding involved. Prioritizations and decisions are being made by senior management. In this way, 152 internal projects were identified between 2014 and 2017 and 128 successfully concluded. ${ }^{7}$ These projects implement sometimes small changes by adding new tasks and reducing others at a local level but the net effect is systemic change in the organization that is related to strategy.

With respect to re-skilling, this approach has the advantage of involving staff directly in the project, elucidating task-specific training and qualification requirements. In many cases, explicit training programs are not necessary because the persons are learning on-the-fly what new skills they have to develop. Additionally, persons usually involved in repetitive service work get involved in project work and learn or improve how to see their work as a continuous change process - an attitude that is often otherwise exclusively nurtured in R\&D departments.

7 https://www.sub.uni-goettingen.de/en/about-us/portrait/strate gy/strategic-goals-and-projects-in-2017.

\section{Task efficiencies and task cancellations}

Sustainability and reliability is at the heart of the reputation of libraries. Any cancellation or even reduction of tasks is therefore to be implemented carefully as it runs against the general mission of a library. However, some tasks can be performed more efficiently or are decreasing. And realism dictates that hard decisions have to make to strategically cut tasks or prefer some over others. For example, print budgets are often cut and invested in digital content licensing, which has consequences for cataloguing, print circulation or cover binding. In this sense, larger parts of libraries already were being changed continuously. An account of finding efficiencies within the organization through cost-neutral change projects has already been described in the previous paragraph. The question of actual task cancellations is much more dependent on external factors. In academic libraries these external factors are determined by the demands of students, researchers and the general public, often correlating to the three missions of a university (Research, Teaching and Public Engagement). Many libraries perform regular surveys on user satisfaction and upcoming demands to better understand external factors. Assessing which tasks can be added or reduced also requires a thorough understanding of the current and prospective service portfolio. Considering the recent pace of transformation, the understanding of the library service portfolios might not always be as trivial as it sounds. Students, researchers and the general public may have a completely different understanding than libraries themselves. For example, libraries are still often seen as book circulation machinery while internally libraries are rather organized as a digital data management operation. Additionally, the radical changes that already have been implemented by libraries (strategically by way of constraint satisfaction), are not always represented in the organizational chart of the library. Accordingly, there is a risk of dissociation between service provision (offer) and service consumption (demand).

In order to communicate and discuss changes in library services, including task cancellations, the SUB undertook an exercise to re-factor the service portfolio. Senior management and staff were asked to define a simple service portfolio that can be understood by researchers, students and the general public. The internal organization of the SUB with its departments and groups was to be disrespected. A maximum of 25 services in maximally 5 service areas were to be defined. A draft result shows the two traditional service areas of "Learning Space" and "Infor- 


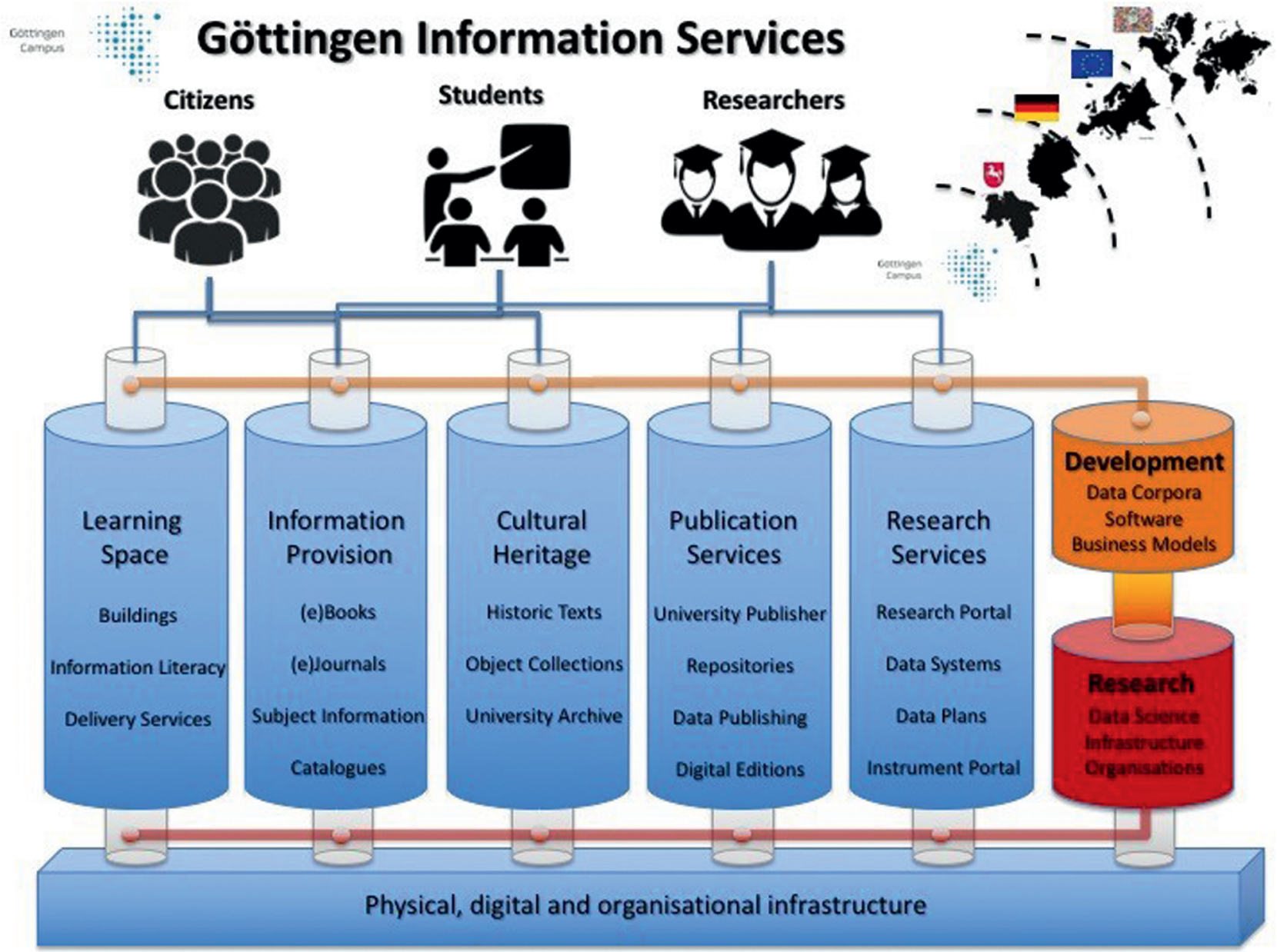

Fig. 4: Functional structure of the SUB, showing the main service areas as well as the innovation pipeline. It is designed to be user-centric and has dimensions beyond the Göttingen Campus, i.e. the state of Lower Saxony, Germany, Europe and international.

mation Provision" (with a third on "Cultural Heritage” that will frequently occur in libraries with special collections). Two novel areas for "Publication Services" and "Research Services" were added (see Figure 4). This division of services also corresponds with the user surveys in 2014 and 2018, which showed demands in exactly those areas. These services shall now be used to discuss possible changes, including cancellations, in the portfolio with researchers, students and other stakeholders. In order to manage change internally, full cost calculations are being developed for steering and monitoring budget allocation.

\section{The role of R\&D}

In the preceding paragraphs, $\mathrm{R} \& \mathrm{D}$ was not treated as a structural element in service transformation. This shall support the transferability of findings to libraries that do not have dedicated R\&D operations. However, R\&D obviously can support service transformation by providing innovation capacity and feeding the pipeline of a dynamic service portfolio management.

At the SUB, R\&D is mainly financed by project funding. Grants from research funders and special grants from the University of Göttingen (state funding from Lower Saxony) became a major factor in the SUB strategy. To support an intuitive comprehension of the situation at the SUB: the extra funding raised from 2012 to 2017 would allow operating the complete SUB for almost two years. Thus, the library, traditionally a service organization based on recurrent funding, has added substantial characteristics of an engineering company and a research department based on project funding. R\&D has specific effects on the capacity to add (and reduce) tasks. First, in certain cases staff appointed for R\&D projects can be seen as a measure to provide bridge-funding for pre-filling (see above). Second, staff appointed for R\&D represents substantial skills increase in the library and can be appointed to permanent positions that are required by service transformation. Third, R\&D projects increase the general project culture in 
the organization and the ability of project management that is needed for service transformation. Fourth, outcomes of the R\&D projects (e.g. software or service requirement specifications) are candidates for becoming a newly added service in the library. In sum, R\&D might not be a necessary condition for service transformation in libraries but R\&D supports service transformation in a systemic way (see also Figure 4).

\section{Discussion}

Service transformation is common practice in academic libraries. One question is whether libraries transform fast enough and whether they have a sufficiently precise model for strategically steering transformation (see above)? In order to get on top of change processes and strategically steer them rather than being subdued to incremental constraint satisfaction, the SUB has taken measures to make transformation an integral part of its organizational culture. The experiences show that certain managerial instruments are essential:

1. Business Intelligence: Appointment capacity, for example, is a crucial but non-trivial factor that requires curated data about staff. Financial plans with costmodels and forecasting are required to steer, control and monitor progress. The SUB introduced a dedicated position for financial controlling.

2. Project Culture: Change is essentially dependent on project management capability and a structured approach to internal and externally funded projects. The SUB introduced a dedicated project office for managing those.

3. Practical Strategy: Strategic plans alone are not sufficient. Implementation plans with specific sometimes complex, sometime simple change projects that are democratically involving staff at all levels are required. The SUB introduced an annual strategic process for this reason.

4. Simple Service Portfolio: Change requires communication and especially the decision making of task cancellations requires that it can be easily explained what a given library does and what is lost, if tasks are cancelled. The SUB has introduced a service-portfoliomanagement model for this.

5. R\&D: Though optional, R\&D fosters change by building capacity in staff and fills the service pipeline. The SUB has a dedicated R\&D department and nurtures the culture of writing project proposals in all parts of the organization.
It can be concluded that these instruments can provide sufficient measures to steer and to determine the pace of service transformation. Whether the pace of transformation is fast enough, however, is a different question. In a retrospective analysis at the SUB, it was found that over the last 25 years roughly 50 positions were introduced to support novel processes (while roughly 25 positions had to be cut). These changes amount to $25 \%$ of staff and, thus, a pace of $1 \%$ transformation per year. Considering that the SUB has a substantial innovation capacity through R\&D projects, maybe not regularly found in other libraries, these results indicate that the pace of transformation in academic libraries is not fast enough. It would take 50 years to transform half of the library operations. Large parts of research, teaching and learning have transformed radically over the last two decades and the transformation is ongoing. Data Science is entering the scene and statistical, analytical or digital qualifications are still not at the heart of library education. It is a valid question, whether it will be sufficient that 50 years from now, in the year 2068, libraries have changed half of their service portfolio, i.e. whether libraries have sufficient transformation capacity to respond to innovations in research, teaching and learning?

Libraries face the dilemma that the transformation of the academic information system is faster than the inherent transformation capacity of libraries. The academic system - and society at large - is facing a singularity, a phase in which organizational cornerstones such as libraries are immersed, and maybe drowned, in a wave of innovation. These considerations suggest that a substantial investment program is needed that helps libraries to transform faster. The analysis above indicates how such an investment program could look like. It should provide the bridge-funding to pre-fill positions, to catch up with innovation and then return to regular operations. For example, in a library with 100 FTE, bridge-funding for 5 FTE a year (50 FTE over 10 years) would already allow to speed up transformation substantially. Implementing the changes in libraries will not be trivial but necessary to maintain libraries as robust and stable pillars of research, innovation and an informed, educated society.

\section{References}

Horstmann, Wolfram (2017): From collecting to connecting - the role of libraries in Open Access. In: Praxishandbuch Open Access, hg. v. Konstanze Söllner und Bernhard Mittermaier. Berlin, Boston: De Gruyter, 62-74. https://doi.org/10.1515/ 9783110494068-008. 
Julien, Heidi; Gross, Melissa; Latham, Don (2018): Survey of Information Literacy Instructional Practices in U. S. Academic Libraries. In: College \& Research Libraries, 79 (2), 179-99. https://doi. org/10.5860/crl.79.2.179.

Kramer, Bianca; Bosman, Jeroen (2016): Innovations in scholarly communication - global survey on research tool usage. F1000Research, 5, 692. https://doi.org/10.12688/ f1000research.8414.1.

Prinsloo, Tanja; Van Deventer, J. P. (2017): Using the Gartner Hype Cycle to Evaluate the Adoption of Emerging Technology Trends in Higher Education - 2013 to 2016. In: Emerging Technologies for Education, ed. by T.-C. Huang, R. Lau, Y.-M. Huang, M. Spaniol, and C.-H. Yuen. (Bd. 10676), 49-57. Cham: Springer International Publishing. https://doi.org/10.1007/978-3-319-710846 6.7.

Tenopir, Carol; Hughes, Dan; Allard, Suzie et al. (2015): Research Data Services in Academic Libraries: Data Intensive Roles for the Future? In: Journal of eScience Librarianship, 4 (2), e1085. https://doi.org/10.7191/jeslib.2015.1085.
Tenopir, Carol; Talja, Sanna; Horstmann, Wolfram; Late, Elina; Hughes, Dan et al. (2017): Research Data Services in European Academic Research Libraries. In: LIBER QUARTERLY, 27 (1), 2344. https://doi.org/10.18352/lq.10180.

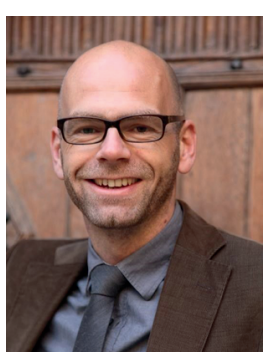

Prof. Dr. Wolfram Horstmann Niedersächsische Staats- und Universitätsbibliothek Göttingen Georg-August-Universität Göttingen Platz der Göttinger Sieben 1 D-37073 Göttingen horstmann@sub.uni-goettingen.de 